\title{
Quadratic residue modulo a power of 2
}

\author{
THINH D. NGUYEN* \\ Moscow State University \\ kosmofarmer@gmail.com
}

July 25, 2018

\begin{abstract}
Quadratic residue modulo an odd prime power has been studied for centuries. Many mathematical tools have been devised to deal with those odd prime power. The left moduli of powers of 2 are thus naturally the subject of this article. We set the objectives of showing some intriguing properties of quadratic residues modulo an even prime power. Though humble in its significance, our results are achieved after years of reading Prasolov's and I. F. Sharygin's maths books.
\end{abstract}

\section{NotATIONS, OBSERVATIONS AND PROPERTIES}

Experimentally, it seems like every $a \equiv 1 \bmod 8$ has 4 square roots modulo $2^{n}$ for all $n \geq 3$. In other words, the equation $x^{2} \equiv a \bmod 2^{n}$ has 4 solutions. We will start by considering 1 as a quadratic residue modulo $2^{n}$. Then, we proceed to generalize the obtained property to arbitrary residue $a$.

\section{i. Number of square roots of 1}

Claim: For all $n \geq 3$, the number of solutions of $x^{2} \equiv 1 \bmod 2^{n}$ is 4 . The solutions are $x \equiv \pm 1 \bmod 2^{n}$ and $x \equiv \pm 1+2^{n-1} \bmod 2^{n}$.

Proof: We want $x^{2}-1 \equiv 0 \bmod 2^{n}$, that is, $(x-1)(x+1) \equiv 0 \bmod 2^{n}$. Since $x$ must be odd, the GCD of $x-1$ and $x+1$ is 2 . Either all of the 2's come from $x-1$, or all the 2's come from $x+1$, or $n-1$ of them come from one $x-1$ or $x+1$, and one of them comes from the other.Q.E.D

\footnotetext{
*Perebor
} 


\section{ii. Square roots of an arbitrary residue}

Observe that $a 2^{t}$ is a quadratic residue where $a$ is odd iff. $t$ is even and $a$ is a quadratic residue modulo $2^{n-t}$. So, in the following, we only consider the case of odd residues. Let $a$ be an arbitrary odd residue modulo $2^{n}$. First, notice that if $u$ and $v$ are two square roots of $a$, we also have $u v^{-1}$ a square root of 1 . Conversely, if $u v^{-1}$ is a square root of $1, u$ and $v$ are square roots of the same residue, i.e. $u^{2}=v^{2}$. As a consequence, if we can find a square root of $a$, we have found 4 square roots of $a$. We can conclude that either $a$ has no square roots i.e. $a$ is a quadratic nonresidue, or $a$ has exactly 4 square roots. Second, a square is necessarily congruent to 1 modulo 8 .

Claim: Restricted to odd residues, the squaring function is $4-$ to -1 and the range is the set of residues congruent to 1 modulo 8 .

Proof: All that is left to prove the claim is that every residue congruent to 8 is a quadratic one.

Indeed, by induction, we can prove the existence of a square root of any residue congruent to 1 modulo 8 . The case $n=3$ is easy to check; if $x^{2} \equiv a \bmod 2^{n}$ then $x^{2}=a+2^{n} k$ for some integer $k$; it is easy to see that $x$ is odd and that

$$
\left(x+2^{n-1} k\right)^{2}=a+2^{n} k(1+x)+2^{n+1}\left(k^{2} 2^{n-3}\right) \equiv a \bmod 2^{n+1}
$$

Q.E.D

\section{REFERENCES}

[1] Michael R. Garey, David S. Johnson, Computers and Intractability: A Guide to the Theory of NP-Completeness

[2] Robertson, N.; Seymour, P. D.; Graph Minors. XIII. The Disjoint Paths Problem, Elsevier Jan. 1995

[3] Phan Dinh Dieu, Le Cong Thanh, Le Tuan Hoa, Average Polynomial Time Complexity of Some NP-Complete Problems. Theor. Comput. Sci. 46(3): 219-237 (1986)

[4] Thinh D. Nguyen, Taking square roots of $\mathbf{- 1}$, https://osf.io/5fmhd/

[5] Thinh D. Nguyen, Taking quartic roots of 2, https://osf.io/wnvaf/ 